\title{
Propagation axiale d'ondes dans une cible cylindrique creuse
}

\author{
F. LÉON, G. MAZE et J. RIPOCHE
}

L.A.U.E., URA 1373 du CNRS, Université du Havre, place Robert Schuman, 76610 Le Havre, France

\begin{abstract}
Acoustic scattering from an isotropic elastic hollow cylindrical shell of infinite length excited by an obliquely incident plane acoustic wave is investigated. Firstly, a Method of Isolation and Identification of Resonances called MIIR in "propagation" enables us to observe the propagation of waves (in a direction parallel to the cylinder axis) like the guided waves $p=2$ and $p=1$. An isolation and an identification are possible in an important range of incidence, contrary to a configuration of two transducers according to Snell-Descartes' law. Secondly, the nature of these waves is analysed with the axial displacement. The choice of this displacemement is confirmed by the angular diagram of the pressure radiated by the circular section, at the end of the tube.
\end{abstract}

Dans l'étude de la diffusion acoustique par des objets insonés, l'application de la M.I.I.R. "classique" [1] (Méthode d'Isolement et d'Identification des Résonances) permet de saisir le processus de diffusion à deux moments privilégiés de son déroulement, afin d'obtenir deux spectres: un spectre de rétrodiffusion et un spectre de résonances. Les résonances, étant isolées, peuvent être identifiées. Dans le cas de l'insonation oblique, cette méthode s'applique avec un positionnement des transducteurs, émetteur et récepteur, en "Snell-Descartes". Le spectre de réflexion et le spectre de résonances d'un tube d'aluminium, caractérisé par son rapport des rayons (b/a) égal à 0,73 , sont obtenus. Néanmoins, au delà d'un angle de $8^{\circ}$, l'isolement des résonances s'avère difficile. Compte tenu de l'effet de réfraction des ondes de surface [2], l'établissement de la résonance se situe en dehors de la zone d'insonation. Il s'avère donc que si l'angle d'incidence, défini par rapport à la normale à l'axe du tube, augmente, la position des transducteurs en "Snell-Descartes" n'est pas adaptée à l'observation des résonances. La solution est alors "d'écarter" les transducteurs, émetteur et récepteur. L'insonation d'une cible cylindrique sur une zone déterminée provoque, aux fréquences de résonance, la propagation axiale d'ondes de familles différentes. La disposition des transducteurs permet, compte tenu des vitesses de propagation axiale des trains d'onde (vitesse de groupe), de sélectionner des familles de résonances liées aux ondes guidées ou aux ondes circonférentielles. La M.I.I.R. utilisée "en propagation" [3] permet donc de tracer, à partir de signaux se propageant le long du tube, des spectres de quasi-raies dont chaque raie est liée à une résonance de la cible, et d'identifier expérimentalement (sur un signal se propageant axialement) le mode de vibration à des angles d'incidence supérieurs à ceux de la 
M.I.I.R. "classique" [1]. Ce procédé permet de relever séparément un spectre de résonances lié aux ondes circonférentielles et un spectre de résonances lié aux ondes guidées, à chaque angle d'incidence [3]. Ce travail porte essentiellement sur l'étude des ondes guidées.

\section{DISCUSSION RELATIVE AUX ONDES GUIDEES $p=1$ ET $p=2$ SOUS INCIDENCE OBLIOUE}

En raison de leur nature, les ondes guidées s'observent dans une gamme d'incidence large. Une étude particulière des ondes guidées $p=1$ et $p=2$ a été réalisée sur un tube d'aluminium de rapport b/a égal à 0,73 . La M.I.I.R. "en propagation" est utilisée; un tel procédé permet alors de s'affranchir de l'effet de l'angle de réfraction, et ainsi de réaliser des identifications à des angles d'incidence grands. Il est mis en évidence que, bien qu'appartenant à des familles d'ondes guidées $(p=1$ et $p=2)$, ces ondes ont un comportement différent en fonction de l'incidence. Les résonances de l'onde guidée $p=1$ sont observables quel que soit le mode sur toute la gamme de fréquence et toute la gamme d'incidence considérées. Par contre, selon le mode, les résonances de l'onde guidée $p=2$ peuvent être présentes à certains angles et absentes à d'autres angles. Le report des points expérimentaux sur les caractéristiques théoriques $\Gamma=f(\alpha)$ (résonances identifiées représentées par ) permet de justifier la non-détection de certaines résonances pour certains angles; Cette valeur $\Gamma$ caractérise la réémission de l'onde de surface dans l'eau, une largeur $\Gamma$ grande signifiant que la résonance ne peut pas se former. Deux cas peuvent se présenter pour les modes de l'onde guidée $p=2$ :

- Un mode donné de résonance est détecté sur toute la gamme d'angle d'incidence étudiée. Cela est confirmé théoriquement par une largeur $\Gamma$ faible dans cette gamme d'incidence, signe qu'une résonance peut se former (Fig.1, mode $n=13$ et 14).

- Un mode donné de résonance est détecté aux faibles angles d'incidence et à de grands angles d'incidence; mais il peut être absent à des angles intermédiaires. Le tracé de la largeur $\Gamma$ de ce mode en fonction de l'angle d'incidence montre une évolution de cette largeur sous forme d'une "cloche". Le sommet de cette

"cloche" correspond à une largeur $\Gamma$ grande; les résonances se forment diffici-

lement à des angles intermédiaires (Fig. 1, mode $n=7$ et 8).

Par ailleurs, dans le cadre de cette étude, à un angle d'incidence fixé et inférieur à $16^{\circ}$, la non-détection de certains modes peut s'expliquer par l'évolution de la largeur $\Gamma$ croissante, puis décroissante lorsque le numéro du mode augmente. Les modes dont la largeur est la plus importante ne sont pas détectés (Fig.2, exemple à un angle d'incidence de $10^{\circ}$ ).

\section{STRUCTURE DU DEPLACEMENT AXIAL ET}

\section{REEMISSION EN BOUT DE TUBE DES ONDES GUIDEES $(p=1$ et $p=2)$}

L'étude expérimentale de la diffusion acoustique par un tube d'aluminium caractérisé par un rapport des rayons de 0,73 a permis de relever divers diagrammes angulaires de la pression rayonnée en bout de tube [4]. La comparaison entre diagrammes angulaires expérimentaux et diagrammes angulaires théoriques de la pression rayonnée en bout de tube montre un excellent accord (Fig.3 et Fig.4). Celui-ci valide le choix du champ élémentaire de déplacement axial $U_{z n}$ (d'indice $n$ correspondant au mode de résonance), ce 
déplacement étant calculé théoriquement. L'analyse de la structure radiale de ce déplacement (Fig.5 et Fig.6) montre que celle-ci est indépendante du mode, mais dépend de la famille de l'onde guidée ( $p=1$ ou $p=2)$. L'analogie entre le déplacement d'une onde de cisaillement $\left(\mathrm{T}_{0}, \mathrm{~T}_{1} \ldots\right)$ sur l'épaisseur d'une plaque et du déplacement axial d'une onde guidée $(p=1, p=2 \ldots)$ sur l'épaisseur du tube a pu être constatée. Dans les deux cas (plaque et tube), il est montré que le nombre de noeuds relatif au déplacement relevé sur l'épaisseur de la plaque ou du tube est caractéristique de la famille de l'onde. Dans le cas du tube, lindice de la famille est défini par la relation: $p=$ nombre de noeuds +1 .

Par ailleurs, il y a un bon accord entre fréquences de résonance des ondes guidées $(p=1$ et $p=2)$ dans un tube et fréquences de résonances des ondes de cisaillement dans une plaque. Ceci est particulièrement net dans le cas d'un tube à coque mince (tube d'aluminium de rapport $b / a=0,9$ ). Cette concordance dans les résultats justifie le choix du modèle de plaque.

\section{CONCLUSION}

L'étude de la diffusion de tubes cylindriques insonés sous incidence oblique à l'aide de la M.I.I.R. "classique" et de la M.I.I.R. "en propagation", et les considérations théoriques associées sur la largeur $\Gamma$ des résonances ont permis d'expliquer les conditions d'apparition des résonances des ondes guidées. Le calcul du déplacement axial, sa représentation par courbes de niveau, la comparaison avec les ondes de cisaillement dans une plaque ont montré que la structure radiale des courbes de niveau donne une information sur la famille $p$ de l'onde. Ce même calcul permet une modélisation expliquant les diagrammes angulaires de diffusion observés en bout de tube.

\section{[1] G. MAZE, J. RIPOCHE,}

"Méthode d'Isolement et d'Identification des Résonances (MIIR) de cylindres et de tubes soumis à une onde acoustique plane dans l'eau",

Rev. Phys. Appl. 18, 319-326, [1983].

[2] J.M. CONOIR, P. REMBERT, O. LENOIR, J.L. IZBICKI, "Relation between surface helical waves and elastic cylinder resonances", J. Acoust. Soc. Am. 93, 1300-1307, [1993]

[3] F. LÉON, F. LECROQ, D. DÉCULTOT, G. MAZE, "Scattering of an obliquely incident acoustic wave by an infinite hollow cylindrical shell", J. Acoust. Soc. Am., 91, 1388-1397, [1992].

[4] F. LÉON, D. DÉCULTOT, F. LECROQ, G. MAZE, "Radiation of a guided wave at the end of the cylindrical shell", 123rd Meeting of the Acoustical Society of America, Salt Lake City, USA, 11-14 mai 1992, J. Acoust. Soc. Am., 91, 2470, [1992]. 


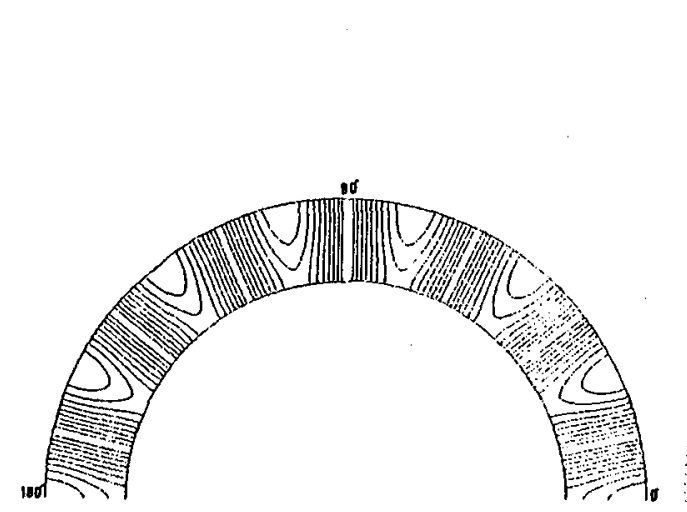

$180^{\circ}$

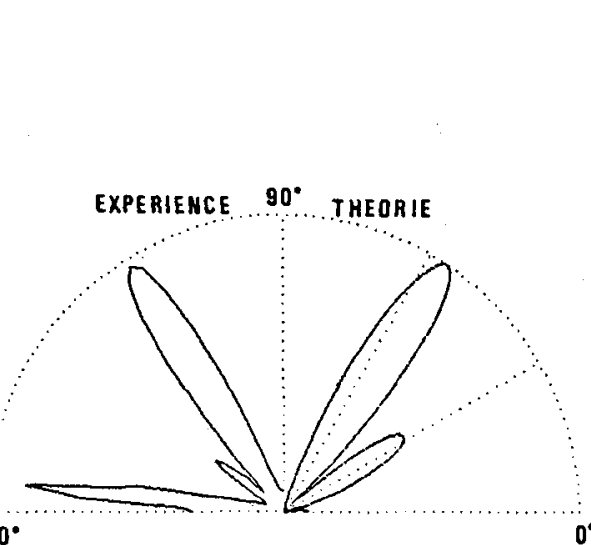

$0^{*}$

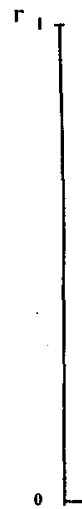

0

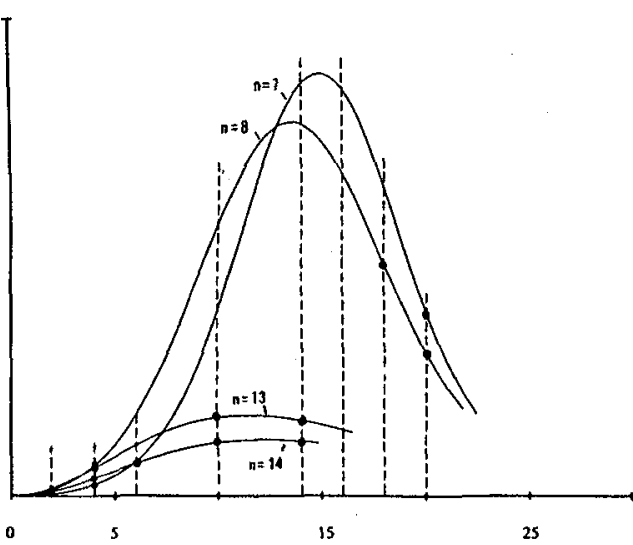

Fig.5 : Représentation sous forme de courbes de Fig. 3 : Diagrammes angulaires théorique et expéri- Fig. 1 : Courbes théoriques $\Gamma=f(\alpha)$, onde de type guidé niveaux du déplacement $\left|U_{m}\right|$.

$\alpha=4^{\circ}$,onde de type guidée $p=1$, $\mathrm{x}_{1}=17,210$, mode $\mathrm{n}=7$.

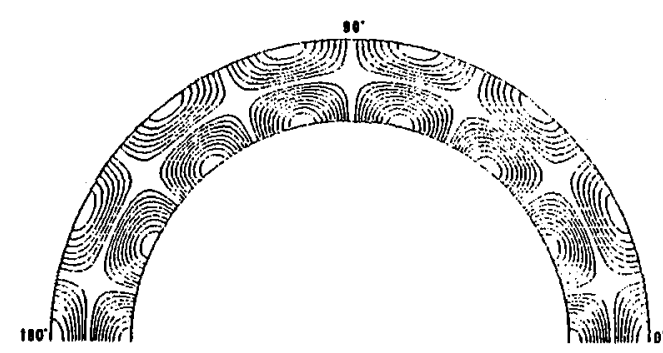

$180^{\circ}$ mental d'une onde guidée $p=1$ de mode 7 . donnée théorique : $x_{1}=17,210$ donnée expérimentale : $x_{1}=17,34$.

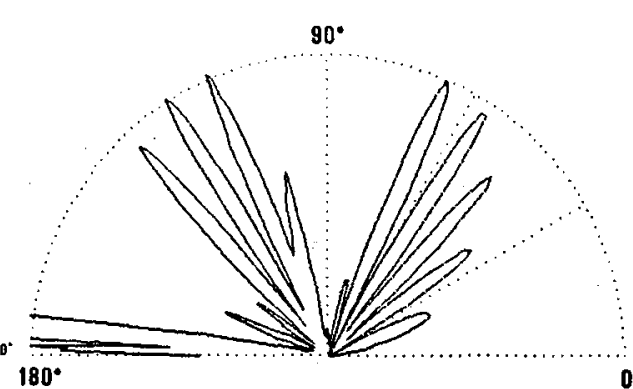

Fig.6 : Représentation sous forme de courbes de Fig.4 : Diagrammes angulaires théorique et expéri- 。 niveaux du déplacement $\left|U_{m}\right|$.

$\alpha=4^{\circ}$, onde de type guidée $p=2$, $x_{1}=30,698$, mode $n=7$.

mentale d'une onde guidée $p=2$ de mode 7 . donnée théorique : $x_{1}=30,698$ donnée expérimentale : $x_{1}=30,19$. $p=2$ et report des résonances identifiées ( $(\bullet)$

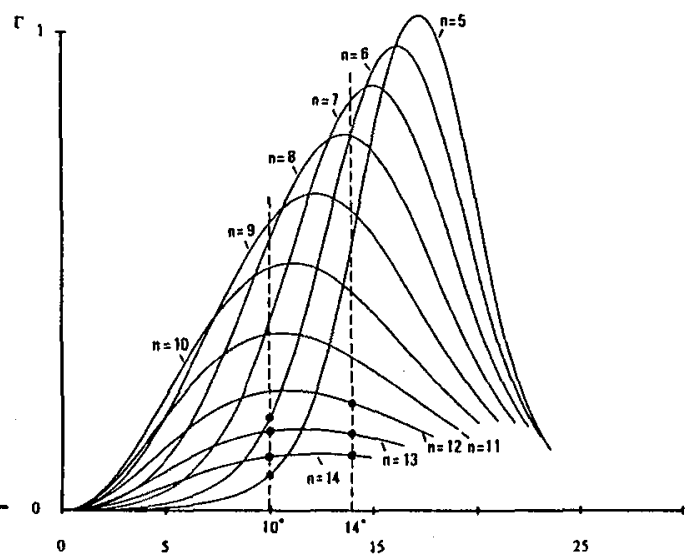

$\alpha\left(0^{\circ}\right)$

Fig. 2: Courbes théoriques $\Gamma=f(\alpha)$, onde de type guidé $p=2$ et report des résonances identifiées $(\bullet)$. 